\title{
Effect of Supplemental Light with Different Spectral LEDs on the Growth of Tomato Seedlings in North Greenhouse
}

\author{
Zou Qiuying $^{1, a}$, JI Jianwei ${ }^{1, b^{*}}$, Li Zhengming ${ }^{1, c}$ \\ ${ }^{1,2,3}$ College of Information and Electrification Engineering, Shenyang Agricultural University, \\ Shenyang 110866, China \\ âE-mail: zouqiuying@126.com, ${ }^{b}$ jianweiji7879@hotmail.com, ' $174887026 @ q q . c o m$,
}

Keywords: LED, Supplemental Light, tomato seedlings, chlorophyll fluorescence parameter. Abstract. The application of light emitting diodes (LEDs) for supplemental lighting is expected to increase quality of tomato seedlings in north greenhouse with less cost. Experiments were carried out to investigate the effect of supplemental lighting by LEDs with two different spectral qualities (blue, red and the combination of the two) on the growth of tomato seedlings. Plants were grown under different spectral light treatments for a month. The photosynthetic photon flux (PPF, 400-700 nm) at the top of plants was adjusted to $100 \mu \mathrm{mol} \mathrm{m}-2 \mathrm{~s}-1$. LEDs were used during the overnight period. The lighting was provided at a PPF level of $50 \mu \mathrm{mol} \mathrm{m}-2 \mathrm{~s}-1$. The air temperatures were kept between 18 and $26^{\circ} \mathrm{C}$. The relative humidity was maintained at $60 \%$ throughout the periods. The results showed that the growth of tomato seedlings was affected by the light quality of the overnight lighting. Particularly, Chlorophyll fluorescence parameters of tomato seedlings in solar greenhouse has change significantly, especially the value of $\varphi_{\text {psII }}$ parameter increased significantly under the combination of red and blue LEDs. It demonstrates that light energy conversion efficiency could be increased in this method.

\section{Introduction}

Plant's leaf absorbs light energy, and part of this is dissipated as heat, another amount is applied for photochemical activity of plant, and a lesser amount is relesed by chlorophyll as lower energy called fluorescence. Chlorophyll fluorescence has been a widely used technique for studies related with the physiology of plant. The application of artificial light for plant growth with light emitting diodes (LEDs) represents a very usefull tool to carry out different studies regarding the effects of the amount and quality. Among various environmental factors, light is one of the most important variables affecting the quality of plant seedlings .

In this paper, based on the parameters of fluorescence, as for the tomato seedlings grown in solar greenhouse of North China, a system for controlling light and detecting photosynthetic ability is proposed. It is necessary to elucidate the most suitable light environment for growing tomato seedling under red and blue light, and to reveal the relation between and light quality and chlorophyll fluorescence in tomato seedlings. To this order, we measured the chlorophyll fluorescence parameters of tomato seedlings grown under a mixture of red and blue to determine the optimum light condition.

\section{Methodology}

The Set-up of Experiment. The experimental setup can be shown in Fig.1. Tomato seedlings were grown in the solar greenhouse of North China. The supplemental lighting system consisted of four parts: array of light-emitting diodes, programmable power supply, which was used to control the light intensity by electricity adjustment, MINIPAM measuring the parameters of chlorophyll fluorescence, and upper PC. LED light source was aligned in a rectangular way, forming the LED set. The wavelength of LED light source was measured by an spectrometer (HR2000, Ocean Optics Inc., FIA, USA). Fig. 1 shows the schematic of supplemental lighting system. 


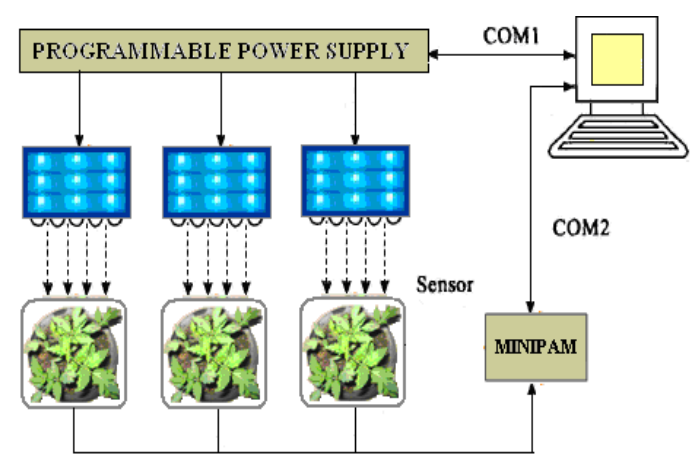

Fig. 1. Schematic of supplemental lighting system

The experiment carried out in greenhouse is shown in Fig.2. The effects of supplemental lighting system on the tomato seedlings was evaluated and analyzed. MINIPAM was used to detect the parameters of chlorophyll fluorescence. The user accesses the supplemental lighting system by an upper PC, through which the desired current can be produced via a man-made software. Then this current was sent to the programmable power supply via RS232 to control the luminous intensity of LED.

Measurement of the photosynthetic efficiency can be derived below: maximum quantum yield of PSII (Fv/Fm=[Fm-F0]/Fm requires dark -adapted leaves) and effective quantum yield of PSII $\left(\varphi p s I I=\left[F m^{\prime}-F s\right] / F m^{\prime}\right.$, requires light-adapted leaves $)$. $\varphi_{\text {psII }}$ can reflect the energy utilization efficiency in photochemistry.

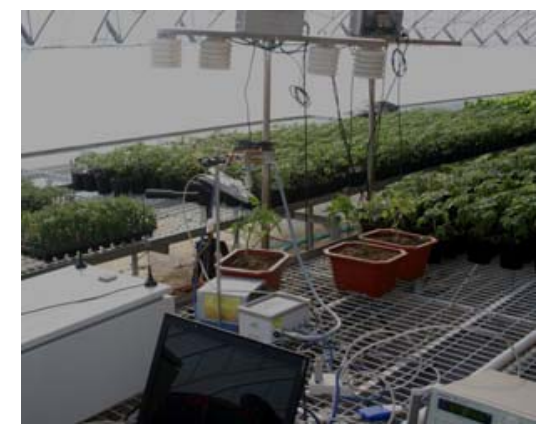

Fig. 2. Experiment under laboratory conditions.

Tomatoes were grown under different spectral light treatments for a month, which in flower bud differentiation stage were irradiated under LED illumination for some time. The photosynthetic photon flux (PPF, 400-700 nm) at the top of plants was adjusted to $100 \mu \mathrm{mol} \mathrm{m}-2 \mathrm{~s}-1$. LEDs were used during the overnight period. The lighting was provided at a PPF level of $50 \mu \mathrm{mol} \mathrm{m}-2 \mathrm{~s}-1$.

\section{Array of Light-emitting Diode (LED)}

Due to the high directivity of LED, the blue and red LED should be arrayed in a proper ratio to achieve a uniform irradiation area. Through simulation and analysis of different modes, the blue and red LED array was determined as Fig.3. There were 36 red LEDs and 9 blue LEDs, and the ratio of it is $4: 1$.

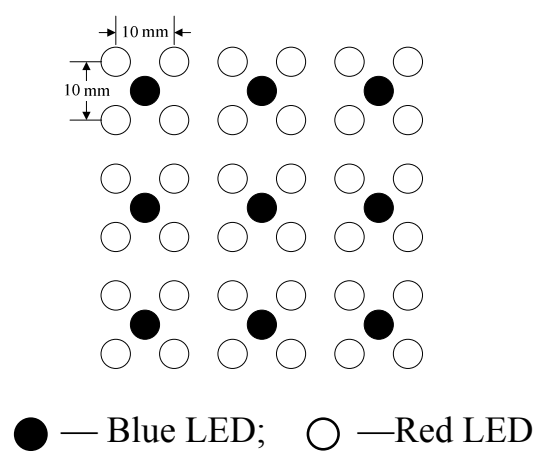

Fig. 3. LED Array 


\section{Control Process}

According to the relationship between the intensity of LED and the fluorescence parameters, the proper ratio of LEDs and illumination cycle was input to the computer. Then physiological analysis of tomato was made based on the dynamic output.The process is shown in Fig.4. Programming language in upper computer is DELPHI 7, SSCOM is a plug-in package of DELPHI for serial communication.

Two-way communication was carried out between MINIPAM and upper PC through RS-232 port. MINIPAM can be controlled based on the Preset command word in upper PC.SQL Server 2000 was applied as the database in development platform. Flow chart of Control was shown in Fig.4.

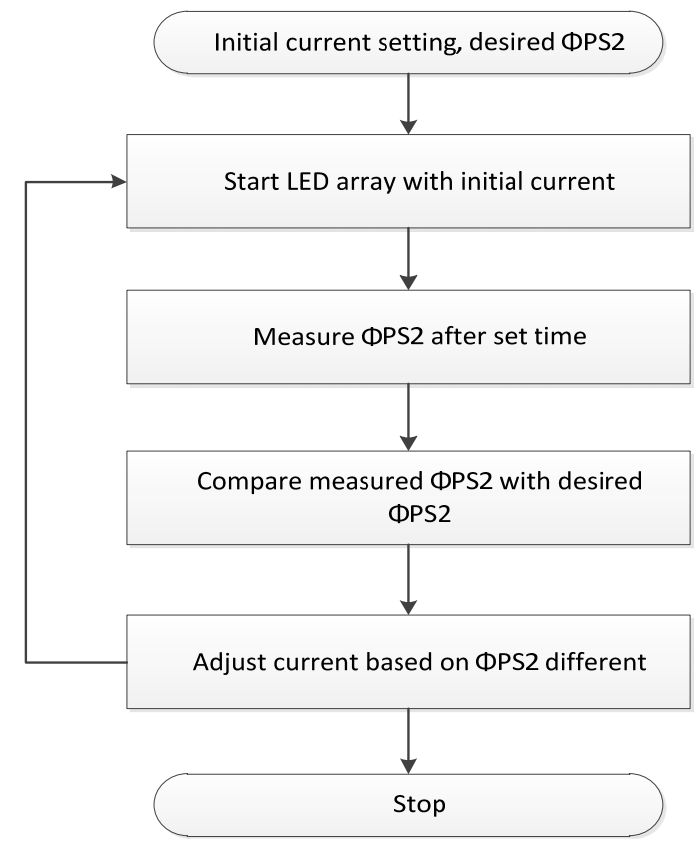

Fig.4 Flow chart of Control

\section{Results and Discussion}

The experiment was carried out in greenhouse (an average day temperature of $26^{\circ} \mathrm{C}$ and a night temperature of $18^{\circ} \mathrm{C}$, the air temperatures were kept between 18 and $26^{\circ} \mathrm{C}$. The relative humidity was maintained at $60 \%$ throughout the periods.). In all chlorophyll fluorescence parameters, $\varphi_{\text {psII }}$ can reflect the photosynthetic rate significantly.

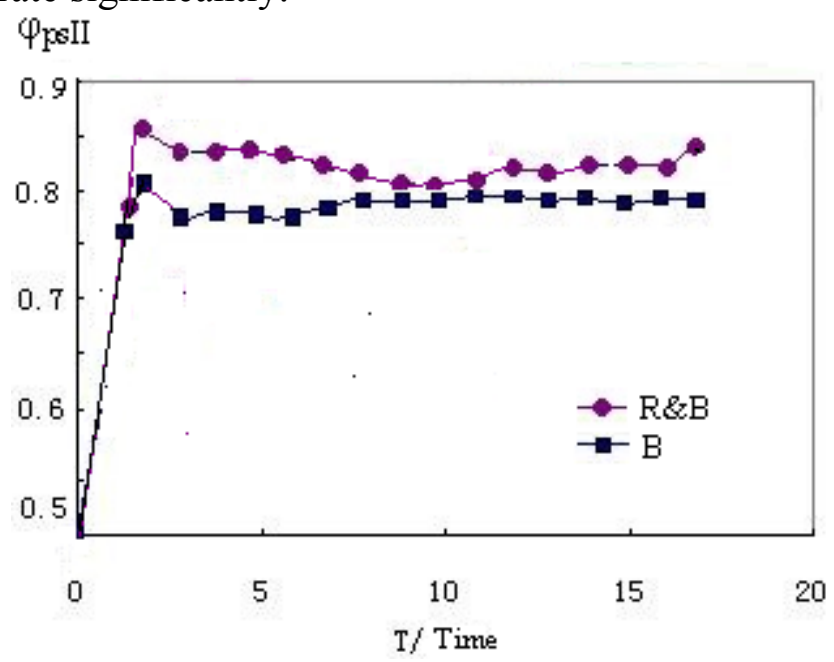

Fig.5 Change of $\varphi_{\text {psII }}$ in different treatment

In Fig.5, the value of $\varphi_{\text {psII }}$ parameter was higher with the combination of red and blue LEDs whose ratio is $4: 1$ than that with only blue LEDs. The curve showed that the system had high 
sensitivity and high accuracy. It can be seen that composite light effect is different from the effect of monochromatic light obviously. The effect is a complex response process, and not the simple accumulation of monochromatic light. This may be the result of the interaction between the spectrum and the special colors of the plant. It is reflected that the open degree of the reaction center of the photosynthetic system is not only relatively larger in the red and blue combined treatment than the single red or blue treatment, but also absorbs and transports more light energy for photosynthesis.

\section{Acknowledgements}

This work was financially supported by Liaoning Provincial Department of Education Project (No. L2012253). The authors would like to acknowledge the Institute of Horticulture, Shenyang Agricultural University, China for their cooperation in performing these experiments in their greenhouse.

\section{References}

[1].Ilieva I., Ivanova T., Naydenov Y., et al., Plant experiments with light-emitting diode module in Svet space greenhouse, Advances in Space Research, 46, 7, 2010, pp. 840-845.

[2].Kolber Z, Klimov D, Ananyev G, Rascher U, Berry J, Osmond B: Measuring photosynthetic parameters at a distance: laser induced fluorescence transient (LIFT) method for remote measurements of photosynthesis in terrestrial vegetation. Photosynthesis Research 2005, 84(1-3):121-129.

[3]. Chen Q-J, Zhang F-M, Wang Y-J, Kurata K: The physiologic reaction of cucumber to low temperature and low light intensigy. Agricultural Sciences in China 2003, 2(2):200-205.

[4] Houter NC, Pons TL: Gap size effects on photoinhibition in understorey saplings in tropical rainforest. Plant Ecology 2005, 179(1):43-51.

[5]. Becker S., Graeve M., Bischof K., Photosynthesis and lipid composition of the Antarctic endemic rhodophyte Palmaria decipiens: effects of changing light and temperature levels, Polar Biology, 33, 7, 2010, pp. 945-955.

[6]. Nichol CJ, Rascher U, Matsubara S, Osmond B: Assessing photosynthetic efficiency in an experimental mangrove canopy using remote sensing and chlorophyll fluorescence Trees 2006, 20(1):9-15

[7]. Gerjo J. Engbers, Influence of spectral light distribution and the photosynthetic acclimation to light of a tomato crop, Wageningen University, 2006.

[8]. Horton P., Hague A., Studies on the induction of chlorophyll fluorescence in isolated barley protoplasts. IV. Resolution of nonphotochernical quenching, Biochim, Biophys. Acta, 932, 1998, pp. 107-115.

[9]. Ralph P. J., Gademann R,. Larkum A. W. D., Spatial heterogeneity in active fluorescence and PSII activity of croal tissues, Mar. Biol, 2002, 141, pp. 539-646.

[10].Lian M., Murthy H. N., Paek K., Effects of light emitting diodes (LEDs) on the in vitro induction and growth of bulblets of Lilium oriental hybrid 'Pesaro', Scientia Horticulturae, 94, 3-4, 2002, pp. 365-370.

[11] Trouwborst G, Schapendonk A H C M, Rappoldt K, et al. The effect of intracanopy lighting on cucumber fruit yield-Model analysis[J]. Scientia Horticulturae, 2011,129(2):273-278.

[12].Genty B., Briantais J. M., Baker N. R., The relationship between the quantum yield of photosynthetic electron transport and quenching of chlorophyll fluorescence, Biochimica Biophysia Acta, 990, 1989, pp. 87-92.

[13].Schreiber U., Bilger W., Neubauer C., Chlorophyll fluorescence as a non-destructive indicator for rapid assessment of in vivo photosynthesis, Ecological Studies, 1, 1994, pp. 49-70.

[14].Peter J. Ralph, Rolf Gademann, Rapid light Curves: A powerfu1 tool to assess photosynthetic activity, Aquatic Botany, 82, 2005, pp. 222-237. 